\title{
SOME FINITELY BASED VARIETIES OF RINGS
}

\author{
Dedicated to the memory of Hanna Neumann
}

TREVOR EVANS

(Received 16 March 1973)

Communicated by M. F. Newman

\section{Introduction}

The results in this paper are consequences of an attempt many years ago to extend to loops some form of the theorem of Lyndon [12] that any nilpotent group has finitely based identities. Having failed in this, we looked for other algebras for which a similar approach might work. The algebra has to belong to a variety in which finitely generated algebras are finitely related and we must be able to bound the number of variables needed in a basis. Commutative Moufang loops, because of the extensive commutator calculus available (Bruck, [4]), provide one example (Evans, [6]). Here we give two examples from rings, namely associative rings satisfying $x^{n}=x$ (more generally, satisfying an identity $x^{2} \cdot p(x)$ $=x$ ) and nilpotent (non-associative) rings. We are also able to extend some results of Higman [9] on product varieties and we show that for associative rings the product of a nilpotent variety and a finitely based bariety is finitely based.

Our result on rings satisfying $x^{n}=x$ has been obtained recently (using a different approach) by Werner and Wille [15] and the nilpotent rings result (in the associative case) is given by Kruse [11] in a paper in which he proves for finite rings the analogue of the Oates-Powell finite basis theorem for the identities of a finite group. However, the elementary nature of our proof for $\left(x^{n}=x\right)$-rings and the extension of Higman's ideas to nilpotent rings may be of interest.

\section{A universal algebra observation}

If a finite algebra with finitary operations is such that its identities are consequences of identities in some bounded number of variables, then its identities

This work was supported in part by an NSF Grant. 
are finitely based (Birkhoff [2], Neumann [14]). The following observation is a mild extension of this result.

LEMMA. Let $\mathfrak{B}$ be a variety of algebras with the property that any finitely generated $\mathfrak{B}$-algebra is finitely presented. Then any subvariety $\mathfrak{U}$ of $\mathfrak{B}$ which can be defined by a set of identities involving a bounded number $k$ of variables is finitely based (relative to $\mathfrak{B}$ ).

Proof. Let $F(\mathfrak{B})$ be the free $\mathfrak{B}$-algebra on a countably infinite set of generators $g_{1}, g_{2}, g_{3}, \cdots$. Let $\theta$ be the fully invariant congruence in $F(\mathfrak{B})$ such that $F(\mathfrak{B}) / \theta$ $\cong F(\mathfrak{U})$, the free $\mathfrak{U}$-algebra on an infinite of number of generators. By hypothesis, $\theta$ may be generated, as a fully invariant congruence, by pairs of words $\left(u, u^{\prime}\right)$ where $u$ and $u^{\prime}$ involve only the generators $g_{1}, g_{2}, \cdots, g_{k}$. Let $F_{k}(\mathfrak{B})$ be the subalgebra of $F(\mathfrak{B})$ generated by $g_{1}, g_{2}, \cdots, g_{k}$ and $\theta_{k}$ the restriction of $\theta$ to $F_{k}(\mathfrak{B})$. Then $\theta$ is generated, as a fully invariant congruence on $F(\mathfrak{B})$, by $\theta_{k}$. Since $F_{k}(\mathfrak{B}) / \theta_{k}$ is a finitely generated $\mathfrak{B}$-algebra, $\theta_{k}$ is a finitely generated congruence on $F_{k}(\mathfrak{B})$. Hence, $\theta$, as a fully invariant congruence on $F(\mathfrak{B})$, may be generated by a finite set of pairs of words in $g_{1}, g_{2}, \cdots, g_{k}$. In other words, $\mathfrak{U}$ may be defined, as a subvariety of $\mathfrak{B}$, by a finite set of identities.

Familiar varieties for which it is known that any finitely generated algebra is finitely related include varieties generated by a finite algebra, abelian groups, commutative semigroups, commutative rings, commutative Moufang loops, nilpotent groups, nilpotent rings. The property fails to hold for groups, rings, lattices and loops.

\section{Identities in rings satisfying $x^{m}=x$}

In this section all rings will be assumed to be associative. The variety of commutative rings satisfies the condition for $\mathfrak{B}$ in the lemma given in Section 2 since a finitely generated commutative ring is finitely related (the Hilbert Basis Theorem). A ring satisfying an identity $x^{n}=x(n>1)$ is commutative (Jacobson, [10]) and we will show that in such a ring any identity is equivalent to a set of identities in at most $n$ variables. Thus the variety generated by the ring satisfies the conditions for $\mathfrak{U}$ in the lemma in Section 2.

THEOREM 1. Any ring satisfying an identity $x^{n}=x(n>1)$ has finitely based identities.

The proof consists of the above remarks and the following lemmas.

LEMMA 1. A ring which satisfies an identity $x^{n}=x$ also satisfies the following identity in $n+1$ variables

$$
\prod\left(x_{i}-x_{j}\right)=0
$$

where the product is taken over all $i, j$ satisfying $1 \leqq i<j \leqq n+1$. 
Proof. We operate in the free commutative ring over the integers generated by $x_{1}, x_{2}, x_{3}, \cdots$. Consider the Vandermonde determinant in $x_{1}, x_{2}, \cdots, x_{n}$

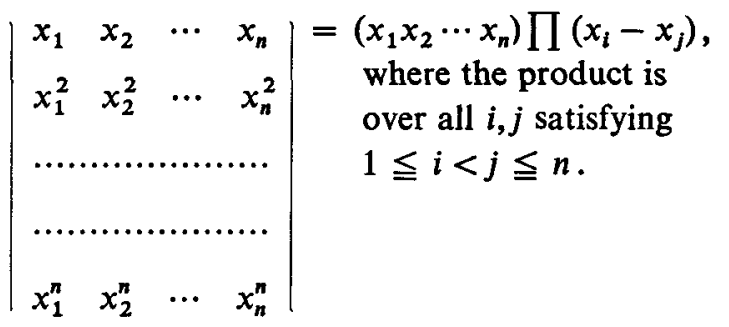

Hence, in any commutative ring satisfying $x^{n}=x$, the identity

$$
\left(x_{1} x_{2} \cdots x_{n}\right) \prod\left(x_{i}-x_{j}\right)=0
$$

will be satisfied. If we write $x_{i}-x_{n+1}$ for $x_{i}$ in this we obtain the identity described in the lemma.

LEMMA 2. Any identity in a ring $R$ satisfying $x^{n}=x$ is equivalent to a finite collection of identities involving at most $n$ variables.

ProOF. We prove the lemma by showing that any identity in a ring satisfying $x^{n}=x$ is equivalent to a collection of identities in at most $n$ variables together with a collection of identities of the form

$$
\left\{\prod\left(x_{i}-x_{j}\right)\right\} w\left(x_{1}, x_{2}, x_{3}, \cdots\right)=0
$$

where the product is over all $i, j$ satisfying $1 \leqq i<j \leqq n+1$. By Lemma 1 , such identities (ii) are consequences of $x^{n}=x$.

Consider an identity $w\left(x_{1}, x_{2}, \cdots, x_{t}\right)=0$ in $t$ variables where $t>n$. Using the Remainder Theorem of elementary algebra to write $w\left(x_{1}, x_{2}, \cdots, x_{t}\right)$ $=\left(x_{i}-x_{j}\right) q\left(x_{1}, x_{2}, \cdots, x_{t}\right)+r\left(x_{1}, x_{2}, \cdots, x_{t}\right)$ where $r$ is $w$ with $x_{i}$ substituted for $x_{j}$, we see that, as an identity in a commutative ring, $w=0$ is equivalent to identities $\left(x_{i}-x_{j}\right) q\left(x_{1}, x_{2}, \cdots, x_{t}\right)=0$ and $r\left(x_{1}, x_{2}, \cdots, x_{t}\right)=0$, where $r=0$ contains fewer than $t$ variables. Now consider an identity in $x_{1}, x_{2}, \cdots, x_{t}$ of the form

$$
\left\{\prod\left(x_{i}-x_{j}\right)\right\} w\left(x_{1}, x_{2}, \cdots, x_{t}\right)=0
$$

where the product is over some pairs $i<j$ in $\{1,2, \cdots, t\}$.

If $x_{p}, x_{q}$ both occur in the left-hand side of (iii) and $x_{p}-x_{q}$ does not, we apply the Remainder Theorem to the left-hand side of (iii) and obtain an identity holding in all commutative rings

(iv) $\left\{\prod\left(x_{i}-x_{j}\right)\right\} w\left(x_{1}, x_{2}, x_{3}, \cdots\right)=\left(x_{p}-x_{q}\right) u\left(x_{1}, x_{2}, x_{3}, \cdots\right)+v\left(x_{1}, x_{2}, x_{3}, \cdots\right)$

where $v$ does not involve $x_{q}$.

Abbreviate (iv) to $A=B+C$. Now multiply both sides by $\left\{\prod\left(x_{i}-x_{j}\right)\right\}^{n-1}$. 
We obtain an identity holding in all commutative rings

$$
\begin{aligned}
\left\{\prod\left(x_{i}-x_{j}\right)\right\}^{n} w\left(x_{1}, x_{2}, x_{3}, \cdots\right)= & \left\{\left(x_{p}-x_{q}\right) \prod\left(x_{i}-x_{j}\right)\right\} u^{\prime}\left(x_{1}, x_{2}, x_{3}, \cdots\right) \\
& +\left\{\prod\left(x_{i}-x_{j}\right)\right\}^{n-1} v\left(x_{1}, x_{2}, x_{3}, \cdots\right)
\end{aligned}
$$

Abbreviate (v) to $A^{\prime}=B^{\prime}+C^{\prime}$. In any commutative ring we will have the following implications between the identities $A=0, B=0, \cdots, C^{\prime}=0$.

$$
\begin{gathered}
A=0 \Leftrightarrow\{(B=0) \quad \text { and }(C=0)\} \\
\Downarrow \\
A^{\prime}=0 \Leftarrow\left\{\left(B^{\prime}=0\right) \text { and }\left(C^{\prime}=0\right)\right\}
\end{gathered}
$$

In a ring satisfying $x^{n}=x$, the identities $A=0$ and $A^{\prime}=0$ are equivalent. Hence, in such a ring the identity $A=0$ is equivalent to the identities $B^{\prime}=0$ and $C=0$. But $B^{\prime}$ has one more linear factor than $A$ in the product and $C$ involves fewer variables than $A$.

If we begin with an identity in $t$ variables in $R$, where $t>n$, by repeating the above procedure we obtain an equivalent collection of identities some of which involve $n$ or fewer variables and the others are of the form (ii). This completes the proof of the lemma and also of the theorem.

ReMARKs. Any commutative ring satisfying a non-trivial monic identity will satisfy a polynomial identity in one variable and hence satisfies the Vandermonde identity $\prod\left(x_{i}-x_{j}\right)=0,1 \leqq i<j \leqq n$, for some $n$. If a ring satisfies an identity $x^{2} p(x)=x$, then it is in fact commutative (Herstein, [8]) and the proof of Theorem 1 may easily be adapted to show that such a ring has finitely based identities. This result is also included in the paper by Werner and Wille [15], mentioned in the introduction. Their proof depends on the lattice of ideals of rings in the variety being distributive and this condition is powerful enough to imply that in any variety of algebras whose lattices of congruences are distributive, the finite algebras have finitely based identities (Baker, [1]).

Although he does not state the theorem explicitly, Cohen [5] has shown (in the course of a study of identities in metabelian groups) that any commutative ring has finitely based identities. I am indebted to R. McKenzie for bringing this to my attention. McKenzie also informs me that he has obtained a different proof of this result. Recently, Bang and Mandelberg [3] have used Cohen's approach to obtain a finite basis theorem for the identities of any ring $R$ (not necessarily associative), such that $R^{n}$ lies on the center of $R$.

\section{Identities in nilpotent rings}

Let $\mathfrak{B}$ be the variety of rings (associativity not assumed). By the nilpotency law of class $c$, (for $c \geqq 1$ ), we mean the set of identities $x_{0} x_{1} x_{2} \cdots x_{c}=0$ where the non-associative product is taken in all possible bracketings. The subvariety of 
$\mathfrak{B}$ defined by the nilpotency laws of class $c$ will be denoted by $\mathfrak{R}_{c}$. A ring is nilpotent of class $c$ if it belongs to $\mathfrak{N}_{c}$ but not to $\mathfrak{N}_{c+1}$.

Let $F$ be the free ring in $\mathfrak{B}$ on a countably infinite set of generators $g_{1}, g_{2}$, $g_{3}, \cdots$ and $F_{k}$ the free $\mathfrak{B}$-ring on $g_{1}, g_{2}, \cdots, g_{k}$. We denote by $F^{(c)}, c=0,1,2, \cdots$ the ideal of $F$ generated by all monomials of length $c+1$ or greater and write $F_{k}^{(c)}$ for $F^{(c)} \cap F_{k}$. Then $F / F^{(c)}$ and $F_{k} / F_{k}^{(c)}$ are free rings in the variety $\mathfrak{R}_{c}$. We note that $F_{k}^{(c)}$ is a finitely generated ideal of $F_{k}$ generated by all momonials of length $c+1$ to $2 c$, inclusive.

If $\mathfrak{B}$ is a subvariety of $\mathfrak{B}$ defined by identities $\left\{u_{i}\left(x_{1}, x_{2}, x_{3}, \cdots\right)=0\right.$; $i=1,2,3, \cdots\}$ and $I$ is the fully invariant ideal of $F$ generated by all values of the $u_{i}$ in $F$, then $F / I$ and $F_{k} / I_{k}$, where $I_{k}=I \cap F_{k}$, are free $\mathfrak{U}$-rings. $\mathfrak{U}$ is a subvariety of $\mathfrak{N}_{c}$ if and only if $I \supseteq F^{(c)}$. The following theorem is equivalent to the statement that any subvariety of $\mathfrak{N}_{c}$ is finitely based.

THEOREM 2. Any nilpotent ring has a finite basis for its identities.

The proof is a consequence of the following lemma, the first of which is a direct analogue of the corresponding theorem for groups (Lyndon, [12]).

Lemma 1. If $S$ is a subring of $F_{k}$, then for each $c=0,1,2,3, \cdots, S$ is generated by a finite set and $S \cap F_{k}^{(c)}$.

Proof. We use induction on $c$. It is trivially true for $c=0$. By the 2 nd isomorphism theorem

$$
S \cap F_{k}^{(c)} / S \cap F_{k}^{(c+1)} \cong\left\{\left(S \cap F_{k}^{(c)}\right), F_{k}^{(c+1)}\right\} / F_{k}^{(c+1)} \subseteq F_{k}^{(c)} / F_{k}^{(c+1)}
$$

and this is a zero-ring which additively is a finitely generated free abelian group. Hence, so is $S \cap F_{k}^{(c)} / S \cap F_{k}^{(c+1)}$. If $S_{i}+\left(S \cap F_{k}^{(c+1)}\right), \quad i=1,2, \cdots, t$ are generators of this additive group, then $S \cap F_{h}^{(c)}$ is generated by the union of $\left\{s_{1}, s_{2}, \cdots, s t\right\}$ and $S \cap F_{k}^{(c+1)}$.

Lemma 2. Any subring of a finitely generated nilpotent ring is finitely generated.

Proof. If $R \in \mathfrak{N}_{c}$, represent $R$ as the quotient ring $F_{k} / I$ where $I \supseteq F_{k}^{(c)}$. If $T$ is a subring of $R$, then $T$ is a homomorphic image of a subring $S$ of $F_{k}$ and by Lemma $1, S$ is generated by a set of elements, all but a finite number of which lie in $F_{k}^{(c)}$.

LEMMA 3. A finitely generated nilpotent ring is finitely related.

Proor. Immediate from Lemma 2. However, we can say more. A finitely generated nilpotent ring is actually finitely presented as a ring since $F_{k}^{(c)}$ is a finitely generated ideal of $F_{k}$.

The theorem will follow from Lemma 3 and the universal algebra observation 
of Section 2 once we have shown that we can bound the number of variables in the identities satisfied by a nilpotent ring. But this is easy to do.

LEMMA 4. The identities of a nilpotent ring of class $c$ are equivalent to a set of identities in at most $c+1$ variables.

Proor. Any ring identity is equivalent to a set of identities, each of which is a sum of monomials which involve the same variables. This follows by appropriate substitutions of zero for variables in the original ring identity. Using the nilpotency laws of class $c$, these sums of monomials may be taken to involve at most $c$ variables. The nilpotency laws of course involve $c+1$ variables.

In order to obtain more information about the identities in a nilpotent ring we give an alternative development leading up to Lemma 4. This approach is similar to that of Higman for nilpotent groups and will enable us to show for nilpotent rings (as Higman did for nilpotent groups) that a finitely generated nilpotent ring satisfies the equation $w\left(x_{1}, x_{2}, x_{3}, \cdots\right)=0$ identically, provided that its elements of "small" length in the generators satisfy it.

Let $\varepsilon_{i}, i=1,2,3, \cdots$ be the endomorphism of $F$ given by $\varepsilon_{i}: g_{i} \rightarrow 0, g_{j} \rightarrow g_{j}$, $j \neq i$. The kernel $K_{i}$ of $\varepsilon_{i}$ is the ideal generated by $g_{i}$ and every element in $K_{i}$ can be written as a sum of monomials each of which has $g_{i}$ as a factor. By a simple induction on $t$, it follows that $K_{1} \cap K_{2} \cap \cdots \cap K_{t}$ is the kernel of $\varepsilon_{1} \varepsilon_{2} \cdots \varepsilon_{t}$ and every non-zero element in it can be written as a sum of monomials each of which involves the generators $g_{1}, g_{2}, \cdots, g_{i}$.

LEMMA 5. Let $w$ be a polynomial in $F$ involving generators $g_{1}, g_{2}, \cdots, g_{t}$. Then $w$ can be written in the form

$$
w=\sum_{i} \pm\left(w \alpha_{i}\right)+w^{*}
$$

where each $\alpha_{i}$ is a product of some of the endomorphisms $\varepsilon_{1}, \varepsilon_{2}, \cdots, \varepsilon_{t}$ and $w^{*}$ is zero or sum of monomials each of which involves the generators $g_{1}, g_{2}, \cdots, g_{t}$.

Proof. This is of course basically a restatement of the proof of Lemma 4 . We may prove it, following Higman [9] by defining $w^{*}=w\left(1-\varepsilon_{1}\right)\left(1-\varepsilon_{2}\right)$ $\cdots\left(1-\varepsilon_{t}\right)$. This is in the kernel of $\varepsilon_{1} \varepsilon_{2} \cdots \varepsilon_{t}$ since $w^{*} \varepsilon_{i}=0$ for each $i$. Hence $w^{*} \in F^{(t-1)}$.

By the length $l(m)$ of a monomial $m\left(g_{1}, g_{2}, g_{3}, \cdots\right)$ in $F$ we mean its length as a groupoid word in the generators and by the length of a polynomial $\Sigma d_{i} m_{i}$, where the $d_{i}$ are integers and the $m_{i}$ are distinct monomials, we mean $\Sigma\left|d_{i}\right| l\left(m_{i}\right)$.

THEOREM 3: If $R$ is a nilpotent ring of class $c$ generated by $a_{1}, a_{2}, a_{3}, \cdots$ and if the equation $w\left(x_{1}, x_{2}, \cdots, x_{k}\right)=0$ is satisfied by all sequences of elements $u_{1}, u_{2}, u_{3}, \cdots, u_{k}$ of $R$ such that $\sum_{i=1}^{k} l\left(u_{i}\right) \leqq c$, then $R$ satisfies $w\left(x_{1}, x_{2}, \cdots, x_{k}\right)=0$ identically. 
Proof. Let $n$ be any positive integer greater than $c$ and assume that $w\left(x_{1}, x_{2}, \cdots, x_{k}\right)=0$ is satisfied by any sequence $u_{1}, u_{2}, \cdots, u_{k}$ of polynomials in $R$ such that $\Sigma l\left(u_{i}\right)<h$. Consider a set of elements $u_{1}, u_{2}, \cdots, u_{k}$ in $R$ such that $\sum l\left(u_{i}\right)=n$.

Let each $u_{i}$ be written as a sum of monomials $\Sigma_{i} \pm m_{i}\left(a_{1}, a_{2}, a_{3}, \cdots\right)$ with coefficients +1 or -1 . Let $\bar{u}_{i}$ be an element of $F$ obtained from $u_{i}$ by replacing each occurrence of each $a_{k}$ in $u_{i}$ by a generator of $F$ so that $\bar{u}_{i}$ is of degree one in each generator. Furthermore, let the sets of generators of $F$ used for different $u_{i}$ be disjoint. Then $\Sigma l\left(\bar{u}_{i}\right)=n$ and $g_{1}, g_{2}, \cdots, g_{n}$ all occur in $w\left(\bar{u}_{1}, \bar{u}_{2}, \cdots, \bar{u}_{k}\right)$.

By Lemma 5 , we can write $w\left(\bar{u}_{1}, \bar{u}_{2}, \cdots, \bar{u}_{k}\right)$ as

$$
\Sigma \pm\left\{w\left(\mathrm{~g}_{1}, \bar{u}_{2}, \cdots, \bar{u}_{k}\right) \alpha\right\}+w^{*}
$$

where $w^{*} \in F^{(c)}$, and each $\alpha$ is an endomorphism of $F$ consisting of a product of endomorphisms $\varepsilon_{i}$. Now $w\left(\bar{u}_{1}, \bar{u}_{2}, \cdots, \bar{u}_{k}\right) \alpha=w\left(\bar{u}_{1} \alpha, \bar{u}_{2} \alpha, \cdots, \bar{u}_{k} \alpha\right)$ and $\sum l\left(\bar{u}_{i} \alpha\right)<n$ since the effect of each $\alpha$ is to replace some generators by zero.

Consider the homomorphism $\beta: F \rightarrow R$ in which for each generator $a_{j}$ of $R$, every generator of $F$ which replaced $a_{j}$ in constructing $\bar{u}_{i}$ from the $u_{i}$ is mapped on $a_{k}$. Then $w\left(\bar{u}_{1}, \bar{u}_{2}, \cdots, \bar{u}_{k}\right) \beta=w\left(u_{1}, u_{2}, \cdots, u_{k}\right)$. But

$$
w\left(\bar{u}_{1}, \bar{u}_{2}, \cdots, \bar{u}_{k}\right) \beta=\Sigma \pm\left\{w\left(\bar{u}_{1} \alpha, \bar{u}_{2} \alpha, \cdots, \bar{u}_{k} \alpha\right\} \beta+w^{*} \beta\right.
$$

and the right-hand side is zero by the combination of our inductive hypotheses and the nilpotence of $R$.

Remarks. Theorem 3 suggests the universal algebraic problem of finding some kind of characterization of varieties for which a similar theorem holds. Of course, for varieties in which finitely generated algebras are finitely related, we can always force a finitely generated algebra to satisfy an identity by imposing a finite set of defining relations consisting of instances of the identity.

\section{Nilpotent by finitely based varieties}

We work in this section entirely within the variety of associative rings and by "ring" we will always mean "associative ring". The results of Section 4 remain valid and we will need in particular Lemma 5 and some of the ideas in the proof of Theorem 3. There is an extensive discussion of products of group varieties in Neumann [14] but since there are some differences on the basic properties of products for group and ring varieties we begin with a summary of these results.

Let $\mathfrak{U}, \mathfrak{B}$ be varieties of rings. The product $\mathfrak{U} \supset \mathfrak{B}$ is the class of all rings which are extensions of a ring in $\mathfrak{U}$ by a ring in $\mathfrak{B}$. That is, $A \in \mathfrak{U} \circ \mathfrak{B}$ if and only if there is an ideal $B \subseteq A$ such that $B \in \mathfrak{U}$ and $A / B \in \mathfrak{B} . \mathfrak{U} \circ \mathfrak{B}$ is a variety since the proof that it is closed under quotients, cartesian products and subalgebras depends only on the isomorphism theorems and is as valid for rings as it is for group 
Let $F$ be the free ring on a countably infinite set of generators $g_{1}, g_{2}, g_{3}, \cdots$. Let $U$ be the fully invariant ideal of $F$ corresponding to the variety $\mathfrak{U}$ and let $\mathfrak{B}$ be the fully invariant ideal of $F$ corresponding to the variety $\mathfrak{B}$. Let $U(V)$ denote the set of elements obtained by substituting for $g_{1}, g_{2}, g_{3}, \cdots$ in any element $u\left(g_{1}, g_{2}, g_{3}, \cdots\right)$ of $U$ any elements $v\left(g_{1}, g_{2}, g_{3}, \cdots\right)$ of $V$. Then $U(V)$ is a fully invariant ideal of $U$. For groups, $U(V)$ is a fully invariant subgroup of $F$ and $F / U(V)$ is free in the variety $\mathfrak{U} \circ \mathfrak{B}$. For rings, all we can say is that $U(V)$ is a fully invariant subring of $F$ and that if $\overline{U(V)}$ is the ideal of $F$ generated by $U(V)$, then $F / \overline{U(V)}$ is free in the variety $\mathfrak{U} \circ \mathfrak{B}$. The difference arises from the fact that if we have rings $A \subseteq B \subseteq C$ where $A$ is a fully invariant ideal in $B$ and $B$ is a fully invariant ideal in $C$, then $A$ is a fully invariant subring of $C$ but not necessarily an ideal. For the same reason, the associative law for the product $\mathfrak{U} \circ \mathfrak{V}$ fails for rings. There are corresponding results for loops. The product of varieties of loops may be defined as for groups but "fully invariant" does not imply "normal" and again the product is not an associative operation (Evans [7]).

However, we can state the following result for rings which is completely analogous to that for groups, and proved in the same way (Neumann, [14]).

Lemma 1. The product $\mathfrak{U} \circ \mathfrak{B}$ of two varieties of rings may be defined by the set of all identities

$$
u_{i}\left(v_{1}\left(x_{11}, x_{12}, x_{13}, \cdots\right), v_{2}\left(x_{21}, x_{22}, x_{23}, \cdots\right), \cdots\right)=0, i=1,2,3, \cdots
$$

where the $u_{i}=0$ are some defining set for $\mathfrak{U}$ and $v_{1}=0, v_{2}=0, \cdots$ run through all identities of $\mathfrak{B}$.

THEOREM 4. Let $\mathfrak{U}$ be a nilpotent variety and $\mathfrak{B}$ a finitely based variety. Then $\mathfrak{U} \circ \mathfrak{B}$ is finitely based.

Proof. $\mathfrak{U}$ may be defined by a single identity $u\left(x_{1}, x_{2}, \cdots, x_{m}\right)=0$ and so by Lemma $1, \mathfrak{U} \circ \mathfrak{B}$ may be defined by the set of all identities

$$
u\left(v_{1}\left(x_{11}, x_{12}, x_{13}, \cdots\right), v_{2}\left(x_{21}, x_{22}, x_{23}, \cdots\right), \cdots\right)=0
$$

where the $v_{l}=0$ run through all identities of $\mathfrak{B}$. Since $\mathfrak{B}$ is finitely based it may be defined by a single identity $v\left(x_{1}, x_{2}, \cdots, x_{n}\right)=0$. If we consider the fully invariant ideal of $F$ generated by $v$ we see that every identity of $\mathfrak{B}$ is of the form $\Sigma_{i} p_{t} v\left(w_{i 1}, w_{i 2}, \cdots, w_{i n}\right) q_{i}=0$ where the $p_{i}, q_{i}$ are monomials and the $w_{i k}$ 's are polynomials in the $x_{i}$ 's. It follows that $\mathfrak{B}$ may be defined by the set of all identities of the form $\sum_{i}^{t} x_{i} v\left(y_{i 1}, y_{i 2}, \cdots, y_{i n}\right) z_{i}=0$ where the $x_{i}, z_{i}, y_{i k}$ are distinct variables, since any identity of $\mathfrak{B}$ may be obtained from one of there by substitution. Similarly, it follows from Lemma 1 , that $\mathfrak{U} \circ \mathfrak{B}$ may be defined by identities of the form 


$$
u\left(\sum_{i=1}^{t_{1}} x_{1 i} v(\cdots) z_{l i}, \sum_{i=1}^{t_{2}} x_{2 i} v(\cdots) z_{2 i}, \cdots, \sum_{i=1}^{t_{n}} x_{n i} v(\cdots) z_{n i}\right)=0
$$

where each argument place in an occurrence of $v(\cdots)$ is occupied by a single variable and distinct blocks of variables are used for the $t_{1}+t_{2}+\cdots+t_{n}$ occurrences of $v(\cdots)$.

There are an infinite number of identities of this form but for any number $t$, only a finite number which involve no more than $t$ occurrences of $v(\cdots)$ since this is equivalent to $t_{1}+t_{2}+\cdots+t_{n} \leqq t$. Let $\mathfrak{U}$ be nilpotent of class $c$ so that it satisfies the identity $x_{0} x_{1} x_{2} \cdots x_{c}=0$. Then $\mathfrak{U} \circ \mathfrak{B}$ will satisfy all identities of the form

$$
x_{0} v(\cdots) x_{1} v(\cdots) x_{2} \cdots x_{c+1} v(\cdots) x_{c} v(\cdots) x_{c+1}=0
$$

where $x_{0}, x_{1}, \cdots, x_{c+1}$ represent either single variables of the empty word and the occurrences of $v(\cdots)$ contain blocks of distinct variables.

The theorem will follow from the next lemma.

LEMMA 2. The identities $\left(^{*}\right)$ are equivalent to the finite set of identities of the same form with $t_{1}+t_{2}+\cdots+t_{n} \leqq c$ together with the finite set of identities $(* *)$.

Proof of Lemma. We use Lemma 5 of Section 3 where we regard the $x_{i j}, z_{i j}$ and the $t v$ 's as generators of a free ring with the $v$ 's corresponding to $g_{1}, g_{2}, \cdots, g_{t}$ in Lemma 5. For $t_{1}+t_{2}+\cdots+t_{n}>c$ in $\left(^{*}\right)$ repeated applications of Lemma 5 yield the result stated.

The idea of the proof is of course exactly the same as that used by Higman to prove the corresponding theorem for groups. We remark that there seem to be difficulties in pushing through this proof for the non-associative case and, in fact, the difficulties are such that it suggests that $\mathfrak{U} \circ \mathfrak{B}$ may not be finitely based if $U$ is non-associative. The finite set of identities $\left({ }^{* *}\right)$ reflecting the nilpotence of $\mathfrak{U}$ needs to be replaced by an infinite set if they are to imply the vanishing of any product involving $c$ occurrences of $v(\cdots)$.

\section{References}

[1] K. A. Baker, 'Equational bases for finite algebras', (Preliminary report), Notices Amer. Math. Soc. 19 (1972), 691-08-2.

[2] G. Birkhoff, 'On the structure of abstract algebras', Proc. Cambr. Philos. Soc. 31 (1935), $433-454$.

[3] C. M. Bang and K. Mandelberg, Finite basis theorems for rings satisfying a central condition (in preparation).

[4] R. H. Bruck, A Survey of Binary Systems, Ergebnisse der Mathematik und ihrer Grenzgebiete vol. 20, (Springer Verlag, 1958).

45] D. E. Cohen, 'On the laws of a metabelian variety', J. of Alg. 5 (1967), 267-273. 
[6] T. Evans, 'Identities and relations in commutative Moufang loops', J. of Algebra (to appear).

[7] $\mathrm{T}$. Evans, Identical relations in loops II (in preparation).

[8] I. N. Herstein, 'The structure of a certain class of rings', Amer. J. Math. 75 (1953), 864-871.

[9] G. Higman, 'Some remarks on varieties of groups', Quart. J. Math. Oxford (2) 10 (1969), 165-178.

[10] N. Jacobson, Structure of Rings, Amer. Math. Soc. Colloq. Publ., v. 37, (Providence, R. I. 1964.)

[11] R. Kruse, 'Identities satisfied by a finite ring', J. of Alg. (to appear).

[12] R. C. Lyndon, 'Two notes on nilpotent groups', Proc. Amer. Math. Soc. 3 (1952), 579-583.

[13] B. H. Neumann, 'Identical relations in groups I', Math. Ann. 114 (1937), 506-525.

[14] Hanna Neumann, Varieties of Groups, Ergebnisse der Mathematik und ihrer Grenzgebiete, vol. 37, (Springer Verlag, 1967.)

[15] H. Werner and R. Wille, 'Charakterisierungen der primitiven Klassen arithmetischner Ringe', Math. Z. 115 (1970), 197-200.

Emory University

Atlanta, Georgia, 30322

U.S.A. 